\title{
Geschlechtergerechtigkeit erfordert mehr! Anmerkungen zur starren Geschlechterquote für Aufsichtsräte
}

\author{
LASSE PÜTZ, MARION WECKES ${ }^{1}$
}

Die Große Koalition will, dass Aufsichtsräte von „voll mitbestimmungspflichtigen und börsennotierten Unternehmen" ab dem Jahr 2016 eine Geschlechterquote von mindestens $30 \%$ aufweisen. ${ }^{2}$ Konkretisiert wird dieses Vorhaben durch den Referentenentwurf eines Gesetzes für die gleichberechtigte Teilhabe von Frauen und Männern an Führungspositionen in der Privatwirtschaft und im öffentlichen Dienst (RefE) vom 09.09.2014, der vom Bundesministerium für Familie, Senioren, Frauen und Jugend sowie vom Bundesministerium der Justiz und für Verbraucherschutz vorgelegt worden ist. ${ }^{3}$ Dieser Entwurf hat bei verschiedensten Gruppen erhebliche Diskussionen ausgelöst. ${ }^{4}$ Die nachfolgenden Ausführungen sollen einen Beitrag leisten, die Diskussion $\mathrm{zu}$ versachlichen.

\section{Gründe für den Referentenentwurf}

In der Einleitung des RefE wird deutlich, dass die beteiligten Ministerien mit der Tatsache, dass der „Anteil weiblicher Führungskräfte in Spitzenpositionen der deutschen Wirtschaft und der Bundesverwaltung [...] nach wie vor gering" ist, unzufrieden sind. Der geringe Frauenanteil widerspreche „einer geschlechtergerechten Teilhabe an verantwor- tungsvollen Positionen in der deutschen Wirtschaft und Verwaltung“ und könne „heute nicht mehr mit deren mangelndem Qualifikationsniveau [...] gerechtfertigt werden“. So sei die Zahl qualifizierter Frauen in Deutschland stetig gestiegen und noch niemals auf einem so hohen Niveau wie heute gewesen. Es sei daher „gesellschaftspolitisch nicht zu erklären, dass Frauen, die über 50 \% der Bevölkerung in Deutschland ausmachen, nach einer gut abgeschlossenen Ausbildung nur zu einem sehr geringen Teil in den Führungspositionen der deutschen Wirtschaft und Verwaltung vertreten sind. ${ }^{\text {"5 }}$

Auch konstatieren die beteiligten Ministerien, dass die in den letzten Jahren von Unternehmen vorangetriebenen freiwilligen Selbstverpflichtungen nicht für mehr Geschlechtergerechtigkeit gesorgt haben und das, obwohl die Bundesregierung bereits im Juli 2001 mit den Spitzenverbänden der deutschen Wirtschaft eine entsprechende Vereinbarung abgeschlossen hatte. ${ }^{6}$ Ebenfalls führten auch die in den Jahren 2009 und 2010 von der Deutsche Corporate Governance Kommission (DCGK) gefassten Kodex-Empfehlungen ${ }^{7}$ zu keiner signifikanten Zunahme von Frauen in Führungspositionen. ${ }^{8}$ Obwohl börsennotierte deutsche Aktiengesellschaften gem. $₫ 161 \mathrm{AktG}$ seitdem verpflich-
1 Die Autoren danken Irene Ehrenstein, Mitarbeiterin im Referat Wirtschaftsrecht der Abteilung Mitbestimmung und Fred Gockeln, Mitarbeiter im Referat Wirtschaft in der Hans-Böckler-Stiftung, für ihre Mitarbeit an diesem Beitrag.

2 Vgl. Koalitionsvertrag (2013): Deutschlands Zukunft gestalten - Koalitionsvertrag zwischen CDU, CSU und SPD für die 18. Legislaturperiode, Abschnitt 4.1.

3 Vgl. RefE (2014): Referentenentwurf eines Gesetzes für die gleichberechtigte Teilhabe von Frauen und Männern an Führungspositionen in der Privatwirtschaft und im öffentlichen Dienst, Bearbeitungsstand 09.09.2014 - 15.03 Uhr.

4 Siehe hierzu beispielsweise: DGB (2014a): Stellungnahme des Deutschen Gewerkschaftsbundes zu dem Referentenentwurf (Bearbeitungsstand 09.09.2014) eines Gesetzes für die gleichberechtigteTeilhabe von Frauen und Männern an Führungspositionen in der Privatwirtschaft und im öffentlichen Dienst; ULA (2014): Stellungnahme des ULA -
Deutscher Führungskräfteverband zum Referentenentwurf eines Gesetzes für die gleichberechtigte Teilhabe von Frauen und Männern an Führungspositionen in der Privatwirtschaft und im öffentlichen Dienst; Bundesvereinigung der Deutschen Arbeitgeberverbände (BDA)/Bundesverband der Deutschen Industrie (BDI) (2014): Geschlechterquote: Richtiges Ziel, falscher Weg, Stellungnahme zum Referentenentwurf eines Gesetzes zur gleichberechtigten Teilhabe von Frauen und Männern an Führungspositionen in der Privatwirtschaft und im öffentlichen Dienst.

5 RefE (2014), a.a.O., S. 1.

6 Ebd., S. 46.

7 Vgl. Deutscher Corporate Governance Kodex (DCGK) (2014), Ziffern 5.4.1 sowie 4.1.5 und 5.1.2, in der Fassung vom 24.06.2014.

8 Vgl. RefE (2014), a.a.O., S. 1 und 46f.; a.A. BDA/BDI (2014), a.a.O. 
tet sind, hierüber jährlich eine Erklärung abzugeben (comply or explain), wurde das Ziel der Kommission, den Anteil von Frauen in Aufsichtsräten ohne eine gesetzliche Regelung zu erhöhen, nicht erreicht. Nach Ansicht der am RefE beteiligten Ministerien sind daher "gesetzliche Regelungen, die den Anteil von Frauen an Führungspositionen in der Privatwirtschaft, in der Bundesverwaltung, in den Unternehmen und Gerichten des Bundes sowie in Gremien im Einflussbereich des Bundes signifikant erhöhen und damit das verfassungsrechtlich verankerte Grundrecht auf gleichberechtigte Teilhabe von Frauen und Männern auch für den Bereich der Führungspositionen erfüllen“, geboten.

\section{Geplante Geschlechterquote im Aufsichtsrat}

Der RefE basiert auf „drei Säulen“. So soll, erstens, ab 2016 eine Geschlechterquote von mindestens 30 \% für Aufsichtsräte gelten; zweitens sieht der Gesetzentwurf vor, dass eine Verpflichtung zur Festlegung von Zielgrößen für Aufsichtsräte, Vorstände und oberste Management-Ebenen besteht. Und drittens ist eine Novellierung der gesetzlichen Regelungen für den öffentlichen Dienst des Bundes beabsichtigt, die im Wesentlichen die Vorgaben für die Privatwirtschaft (Säulen eins und zwei) widerspiegeln soll. Um den Untersuchungsumfang zu begrenzen und auf die Mitbestimmung im Aufsichtsrat zu fokussieren, wird nachfolgend jedoch ausschließlich auf die „erste Säule“ - die Geschlechterquote für den Aufsichtsrat - eingegangen.

Rechtstechnisch soll die Quote von $30 \%$ vor allem durch eine gesetzliche Ergänzung erreicht werden. So sieht der RefE in Art. 3 vor, dass nach Abs. 1 des jetzigen $₫ 96$ AktG u. a. folgender neuer Absatz eingefügt wird:

„Bei börsennotierten Gesellschaften, für die das Mitbestimmungsgesetz, das Montan-Mitbestimmungsgesetz oder das Mitbestimmungsergänzungsgesetz gilt, setzt sich der Aufsichtsrat zu mindestens 30 Prozent aus Frauen und zu mindestens 30 Prozent aus Männern zusammen. Der Mindestanteil ist auf der Seite der Aktionäre und der Arbeitnehmer gesondert zu erfüllen. Es ist zur nächsten vollen Personenzahl aufzurunden. Eine Wahl der Mitglieder des Aufsichtsrats durch die Hauptversammlung und eine Entsendung in den Aufsichtsrat unter Verstoß gegen das Mindestanteilsgebot ist nichtig. Auf die Wahl der Aufsichtsratsmitglieder der Arbeitnehmer sind die in Satz 1 genannten Gesetze zur Mitbestimmung anzuwenden."

Der RefE normiert folglich eine fixe Mindestquote für beide Geschlechter, wenn das betreffende Unternehmen börsennotiert ist und kumulativ einer paritätischen Mitbestimmung nach dem Gesetz über die Mitbestimmung der Arbeitnehmer (MitbestG), dem Montan-Mitbestimmungsgesetz (Montan-MitbestG) oder dem Montan-Mitbestimmungsergänzungsgesetz (MitbestErgG) unterliegt. Ausgenommen von der zwingenden Quote wären damit Unternehmen, die zwar börsennotiert sind, aber die Rechtsform der Europäischen Aktiengesellschaft (SE) aufweisen. In diesem Unternehmen gilt die Mitbestimmung im Auf- sichtsrat aufgrund einer Vereinbarung oder bei Anwendung der Auffangregelung nach dem SE-Beteiligungsgesetz (SEBG), sodass die zitierten Mitbestimmungsgesetze keine Anwendung finden. Gleiches gilt für börsennotierte Unternehmen, die aus einer grenzüberschreitenden Verschmelzung hervorgehen und die auf der Grundlage des Gesetzes über die Mitbestimmung der Arbeitnehmer bei einer grenzüberschreitenden Verschmelzung (MgVG) paritätisch mitbestimmt sind. Für beide Fälle sieht der RefE lediglich eine Soll-Vorschrift vor. ${ }^{10}$

Gelten soll die vorgeschlagene Quote von $30 \%$ ab dem 01.01.2016, wobei Anteilseigner- und Arbeitnehmervertreter $^{11}$ die Quote jeweils gesondert erfüllen müssen und bestehende Mandate bis zu ihrem regulären Ende wahrgenommen werden können. ${ }^{12}$ Es ist daher bezüglich des Wahlverfahrens auch eine gesonderte Betrachtung erforderlich:

(1) Das geplante Gesetz erklärt Wahlen der Anteilseignervertreter (en bloc oder einzeln) für nichtig, wenn sie nicht der Quote entsprechen. Der Gewählte bzw. Entsandte wird damit von Anfang an kein Aufsichtsratsmitglied. Damit diese Sanktion des „leeren Stuhls“ nicht eintritt, ist es daher sinnvoll und vom Gesetzgeber beabsichtigt, die Vorschläge, die der Hauptversammlung gemacht werden, vorab auf ihre "Quotentauglichkeit“ zu überprüfen. ${ }^{13}$

(2) Die detaillierten Regelungen, die aufgrund der Geschlechterquote von $30 \%$ für die Arbeitnehmervertreter bei der Wahl zu beachten sind, und die Frage, wie sich die Nichterreichung der Quote auswirkt, werden nicht im AktG geregelt. Dieses erfolgt vielmehr "systemgerecht in den Mitbestimmungsgesetzen." Dies ist u.a. erforderlich, weil berücksichtigt werden muss, „dass die Besetzung der Arbeitnehmerbank in den drei Mitbestimmungsgesetzen nicht nach einem einheitlichen Verfahren erfolgt. ${ }^{\text {(14 }}$ Deshalb sind hier unterschiedliche Lösungen notwendig, die den jeweiligen Wahlverfahren gerecht werden.

Mit Blick auf die Wahl der Arbeitnehmervertreter in den Aufsichtsrat stellt sich zunächst die Frage, ob die 30 \%-Quote erreicht wird. Geht man davon aus, dass die Arbeitnehmer im Aufsichtsart unabhängig von den Anteilseignervertre-

9 RefE (2014), a.a.o., S. $1 f$.

10 Ebd., Art. 3 sowie Art. 11 und S. 50 ff. sowie S. 101.

11 Nachfolgend werden aus Gründen besserer Lesbarkeit nur männliche Formen verwendet, gemeint sind dabei jeweils beide Geschlechter.

12 RefE (2014), a.a.O., S. 50.

13 Ebd., S. 50 und S. 102.

14 Ebd., S. 50 
tern die Quote von $30 \%$ erfüllen müssen, und gemäß RefE immer aufzurunden ist (mindestens $30 \%$ ), so ergibt sich die in Übersicht 1 (linke Spalte) dargestellte Personenzahl, die beide Geschlechter mindestens auf Seiten der Arbeitnehmer zu erreichen haben.

Für den Fall, dass die erforderliche Personenzahl zur Erfüllung der Quote nicht erreicht wird, sieht der Gesetzentwurf für das MitbestG und das MitbestErgG in einem zweiten Schritt das gleiche Sanktionsinstrument wie bei den Aktionärsvertretern, also den vorübergehend „leeren Stuhl“, vor. Anders jedoch als bei der Wahl der Hauptversammlung oder der Bestellung gemäß $₫ 101$ AktG bedarf es bei der Festlegung, welcher Stuhl frei bleibt, eines deutlich differenzierteren Verfahrens. Dies liegt darin begründet, dass die Arbeitnehmervertreter sich aus unterschiedlichen „Gruppen“ (den Unternehmen angehörende Arbeitnehmervertreter, Vertreter der Gewerkschaften sowie der Leitenden Angestellten) zusammensetzen. Der RefE sieht daher ein Verfahren vor, das zwischen Gewerkschaftsvertretern und betrieblichen Vertretern unterscheidet. Die Position des leitenden Angestellten wird, da es sich immer nur um eine Person handelt, keiner Quote unterworfen. ${ }^{15}$ Für Wahlen nach MitbestG und MitbestErgG zeigt Übersicht 2 die erforderlichen Mindestpersonenzahlen in den einzelnen „Gruppen“. Die Vertreter der Gewerkschaften haben also z. B. bei einem mit zwölf, 15 oder 16 Personen besetzten Aufsichtsrat eine Quote von 50 statt $30 \%$ zu erfüllen. Bei den betrieblichen Aufsichtsratsmitgliedern der Arbeitnehmer beträgt die geforderte Quote in einem Aufsichtsrat mit 16 Personen immerhin noch 40 anstatt $30 \%$.

Wird die jeweilige Mindestpersonenzahl beider Geschlechter für eine Gruppe nicht erreicht, so ist die Wahl der Person, die dem Mehrheitsgeschlecht angehört und die geringsten Stimmen hat, nichtig. In diesem Fall sieht der RefE vor, dass dieser freie Platz neben der Nachwahl auch durch eine gerichtliche Ersatzbestellung nach $\$ 104$ des AktG besetzt werden kann. ${ }^{16}$ In diesem Zusammenhang ist
ÜBERSICHT 1

Geschlechterquotierung der Arbeitnehmervertreter im Aufsichtsrat*

\begin{tabular}{l|ccc}
\hline & $\begin{array}{c}\text { Größe des } \\
\text { Aufsichtsrats }\end{array}$ & $\begin{array}{c}\text { Anzahl der } \\
\text { Arbeitnehmervertreter }\end{array}$ & 30 \% Quote \\
\hline MitbestG & 12 Mitglieder & 6 Mitglieder & 2 Mitglieder \\
& 16 Mitglieder & 8 Mitglieder & 3 Mitglieder \\
& 20 Mitglieder & 10 Mitglieder & 3 Mitglieder \\
\hline Montan-MitbestG & 11 Mitglieder & 5 Mitglieder & 2 Mitglieder \\
& 15 Mitglieder & 7 Mitglieder & 3 Mitglieder \\
& 21 Mitglieder & 10 Mitglieder & 3 Mitglieder \\
\hline Mitbest-ErgG & 15 Mitglieder & 7 Mitglieder & 3 Mitglieder \\
& 21 Mitglieder & 10 Mitglieder & 3 Mitglieder \\
\hline
\end{tabular}

*Iaut RefE (2014), a.a.O.

Quelle: Darstellung der Autoren, angelehnt an RefE (2014), a.a.O, S. 51

bemerkenswert, dass nach Ansicht der Ministerien bei einer Ersatzbestellung des Gerichts nach $₫ 104$ AktG grundsätzlich die „Auswahlfreiheit des Gerichts [...] begrenzt“ sei und aus diesem Grund das Gericht immer „erst [...] den ihm vorgeschlagenen Bewerber des „passenden“ Geschlechts berücksichtigen [sollte], der bei der vorausgegangenen Aufsichtsratswahl bei einer Verhältniswahl auf dem Wahlvorschlag kandidierte, dessen Bewerber nur wegen des „nicht passenden“ Geschlechts nicht wirksam gewählt werden konnte. ${ }^{“ 17}$ Dieses gebundene Ermessen findet sich

15 Vgl. RefE (2014), a.a.O., S. 52.

16 Vgl. RefE (2014), a.a.O., S. 52; der DGB (DGB 2014a, a.a.O.) wie auch die Arbeitgeberverbände (BDA/BDI 2014, a.a.O.) stehen diesem Verfahren kritisch gegenüber.

17 Vgl. RefE (2014), a.a.O., S. 110.

ÜBERSICHT 2

\section{Geschlechtergruppierung im Aufsichtsrat nach Gruppen*}

\begin{tabular}{|c|c|c|c|c|c|c|c|}
\hline & \multirow[t]{2}{*}{$\begin{array}{c}\text { Größe des } \\
\text { Aufsichtsrats }\end{array}$} & \multicolumn{2}{|c|}{$\begin{array}{c}\text { Betriebliche } \\
\text { Arbeitnehmervertreter }\end{array}$} & \multicolumn{2}{|c|}{ Gewerkschaftsvertreter } & \multicolumn{2}{|c|}{$\begin{array}{c}\text { Vertreter der } \\
\text { Leitenden Angestellten }\end{array}$} \\
\hline & & Personenzahl & $\begin{array}{l}\text { Geschlechter- } \\
\text { quote erfüllt bei: }\end{array}$ & Personenzahl & $\begin{array}{l}\text { Geschlechter- } \\
\text { quote erfüllt bei: }\end{array}$ & Personenzahl & $\begin{array}{l}\text { Geschlechter- } \\
\text { quote erfüllt bei: }\end{array}$ \\
\hline \multirow[t]{3}{*}{ MitbestG } & 12 Mitglieder & 3 & 1 & 2 & 1 & 1 & - \\
\hline & 16 Mitglieder & 5 & 2 & 2 & 1 & 1 & - \\
\hline & 20 Mitglieder & 6 & 2 & 3 & 1 & 1 & - \\
\hline \multirow[t]{2}{*}{ Mitbest-ErgG } & 15 Mitglieder & 5 & 2 & 2 & 1 & & \\
\hline & 21 Mitglieder & 7 & 2 & 3 & 1 & & \\
\hline
\end{tabular}

*laut RefE (2014), a.a.O. 
ABB. 1

\section{Frauen im Aufsichtsrat der vom RefE* erfassten Unternehmen 2013}

Frauen

Männer

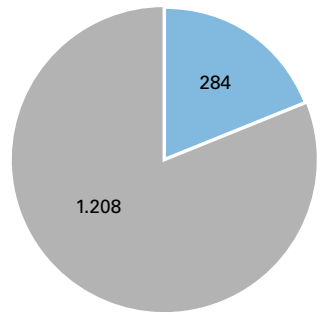

*Referentenentwurf eines Gesetzes für die gleichberechtigte Teilhabe von Frauen und Männern an Führungspositionen in der Privatwirtschaft und im öffentlichen Dienst.

Quelle: Mitbestimmungsdatenbank der Hans-Böckler-Stiftung; Darstellung der Autoren.
Für die Unternehmen, welche unter den Anwendungsbereich der Montan-MitbestG fallen, erfolgt die Sicherstellung der Quote auf die Weise, dass dem Wahlorgan von den Betriebsräten nur ein quotengerechter Vorschlag vorgelegt werden kann. ${ }^{18}$

\section{Fakten zur Geschlechterquote im Aufsichtsrat}

Zum Stichtag 31.12.2013 hätte die im RefE geplante Geschlechterquote von mindestens $30 \%$ eines Geschlechts im Aufsichtsrat 101 Unternehmen erfasst. ${ }^{19} \mathrm{Da}$ in diesen 101 Unternehmen insgesamt 1.492 Mandate zur Verfügung stehen, wäre das Quorum von 30 \% bei 448 Mandaten erreicht. Da jedoch der RefE, wie gezeigt, zwischen Anteilseignerund Arbeitnehmerseite unterscheidet und immer auf die nächste volle Personenzahl aufgerundet wird, erhöht sich die von einem Geschlecht zu erreichende Mindestmandatszahl bei den 101 Unternehmen auf 492 Mandate. In diesen Unternehmen wurden jedoch zum Stichtag 31.12.2013 nur 284 Mandate von Frauen ausgeübt (Abbildung 1).

Folglich hätten zum Stichtag 31.12.2013 rein rechnerisch aber nicht im Gesetzestext, sondern nur in der Begründung. Dies verwundert, weil die Ermessensreduzierung mit demokratischer Legitimation der ersatzbestellten Person und dem hieraus resultierenden großen Rückhalt bei den Wählern begründet wird und daher eigentlich im Gesetzestext normiert sein sollte.

ABB. 2

\section{Geschlechterquote in voll mitbestimmungspflichtigen und börsennotierten Unternehmen}

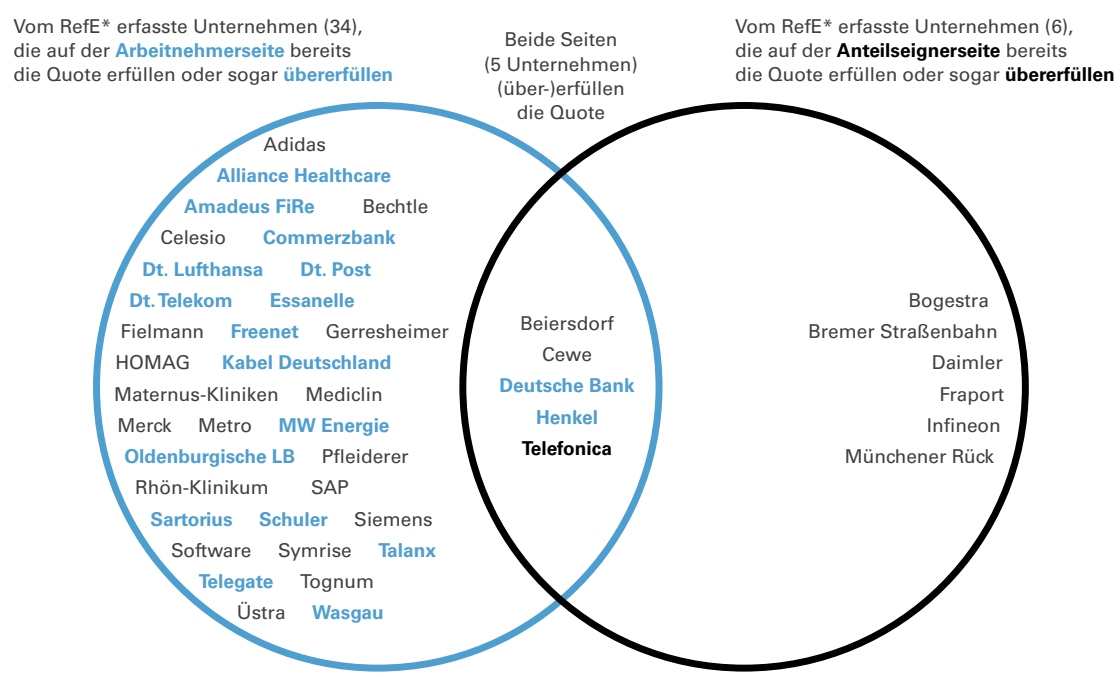

*Referentenentwurf eines Gesetzes für die gleichberechtigte Teilhabe von Frauen und Männern an Führungspositionen in der Privatwirtschaft und im öffentlichen Dienst.

Quelle: Mitbestimmungsdatenbank der Hans-Böckler-Stiftung; Darstellung der Autoren. 208 Mandate mit Frauen statt mit Männern besetzt sein müssen. Da jedoch in einigen Unternehmen bereits heute, überwiegend auf der Arbeitnehmervertreterseite, die geforderte Geschlechterquote übererfüllt wird, kann die Zahl von 208 Mandaten nur ein Anhaltspunkt sein.

Betrachtet man die Verteilung von Frauen im Aufsichtsrat detailliert (Abbildung 2), zeigt sich, dass die Arbeitnehmervertreter in den vergangenen Jahren deutlich mehr für die Geschlechtergerechtigkeit getan haben als die Anteilseignerseite. Nur fünf der vom RefE erfassten 101 Unternehmen erfüllen bereits zum Untersuchungsstichtag die vorgeschlagene Quote auf beiden Aufsichtsratsbänken. Zwei davon übererfüllen die Quote dank der Arbeitnehmervertreter, eins aufgrund der Besetzung der Anteilseignervertreter. Insgesamt zeigt sich aber deutlich, dass die Quotenerfüllung bereits überwiegend auf Arbeitnehmervertreterseite erfolgt ist (39 Unternehmen) und sich darunter sogar 18 Unternehmen befinden, die mehr Frauen auf der Arbeitnehmerbank haben als gesetzlich notwendig wäre. Der Nachholbedarf besteht hier auf Anteilseignerseite: nur elf Unternehmen erfüllen die Quote, eins davon hat lediglich eine Frau mehr als zwingend notwendig wäre. Die Mehrheit der Unternehmen (56) jedoch hat Nachholbedarf auf beiden Aufsichtsratsbänken, wie Abbildung 3 zeigt.

Dabei kann man die Unternehmen jedoch nochmals differenzieren. Unter den genannten 56 Unternehmen befinden sich nämlich nur vier, die keine Frau im Aufsichtsrat

18 Vgl. ebd., Art. 6.

19 Vgl. Pütz, L./Weckes, M. (2014): Geschlechterquote - Geschlechterquote für mehr Frauen in den Aufsichtsräten vor allem Anteilseigner sind gefordert, Mitbestimmungsförderung Report 1-2014, http://www.boeckler.de/pdf/ p_mbf_report_2014_01.pdf (Stand: 16.10.2014). 
haben. Bei der Mehrheit der Unternehmen (23 Unternehmen) gibt es keine Frau auf der Anteilseignerseite. Lediglich neun Unternehmen hatten bis zum Stichtag 31.12.2013 keine Frau auf der Arbeitnehmerseite.

\section{Bewertung des Referentenentwurfs}

Das Vorhaben der Großen Koalition, mehr Geschlechtergerechtigkeit durch eine gesetzliche Quotenregelung zu schaffen, ist angesichts der hier aufgezeigten Fakten und der Entwicklung in den letzten Jahren gerechtfertigt. Weder freiwillige Selbstverpflichtungen der Unternehmen bzw. der Wirtschaft noch die Empfehlung des Deutschen Corporate Governance Kodex haben signifikant mehr Frauen in die Aufsichtsräte und Vorstände gebracht. ${ }^{20}$ Zwar ist die Frage zu stellen, ob das verfolgte gesellschaftspolitische Ziel, wirklich durch eine Novellierung des Gesellschaftsrechts durchgesetzt werden sollte. Letztlich ist der Gesetzgeber jedoch bei der Wahl seiner legislatorischen Mittel weitgehend frei, sodass auch das Gesellschaftsrecht für gesellschaftspolitische Ziele geändert werden kann. ${ }^{21}$

Auch zeigt sich, dass die Umsetzung der Quotenregelung zumindest mittelfristig keine unlösbaren Probleme verursachen würde. ${ }^{22}$ So ist die Anzahl der Mandate, die mit Frauen ab $2016 \mathrm{zu}$ besetzen wäre, gering. Da theoretisch die Anteilseigner jede Frau über 18 Jahren in einen Aufsichtsrat wählen können, ist die Ablehnung der Quote durch die Wirtschaft scheinbar vor allem dem Umstand geschuldet, dass für ein Aufsichtsratsmandat immer noch allein unternehmerische Erfahrung als Vorstand vorausgesetzt wird. Insoweit muss richtigerweise ein ganzheitliches Umdenken zur Geschlechtergerechtigkeit einsetzen.

Jedoch sollte nicht übersehen werden, dass der bisherige Gesetzesvorschlag auch Schwächen hat. ${ }^{23}$ So ist die Reduzierung auf Unternehmen, für die das Mitbestimmungsgesetz, das Montan-Mitbestimmungsgesetz oder das Mitbestimmungsergänzungsgesetz gilt - also die Unterneh-
ABB. 3

\section{Voll mitbestimmungspflichtige und börsennotierte Unternehmen, die die Geschlechterquote weder auf Anteilseigner- noch auf Arbeitnehmerseite erfüllen}

- Arbeitnehmerseite

- Anteilseignerseite

* Referentenentwurf eines Gesetzes für die gleichberechtigte Teilhabe von Frauen und Männern an Führungspositionen in der Privatwirtschaft und im öffentlichen Dienst.

Quelle: Mitbestimmungsdatenbank der Hans-Böckler-Stiftung; Darstellung der Autoren.

men die „voll mitbestimmt“ sind -, nicht nachvollziehbar. Eine Eingrenzung des Anwendungsbereichs aufgrund einer Beschäftigtenzahl (z. B. 2.000 Arbeitnehmer) wäre plausibler. ${ }^{24}$ Unberücksichtigt bleibt auch, dass sich die Arbeitnehmervertreterinnen überwiegend aus der Belegschaft rekrutieren und daher eine Ungleichbehandlung ge-

20 Vgl. Knoll, L./Lochner, D.: Diskriminierung durch Quote?, in: Der Betrieb 67 (10), S. 495-499 (S. 497).

21 Siehe zur umstrittenen Rechts-/Verfassungsmäßigkeit einer (starren) Geschlechterquote nur: BDA/BDI (2014), a.a.O., S. 5; DAV (2014): Stellungnahme des Deutschen Anwaltvereins durch den Arbeitsrechtsausschuss, den Genderausschuss, den Handelsrechtsauschuss und den Verwaltungsrechtsausschuss zum Referentenentwurf eines Gesetzes für die gleichberechtigte Teilhabe von Frauen und Männern an Führungspositionen in der Privatwirtschaft und im öffentlichen Dienst, S. 10f.; Waas, B. (2012): Geschlechterquoten für die Besetzung der Leitungsgremien von Unternehmen - Bewertung der aktuellen Entwürfe aus unionsrechtlicher und rechtsvergleichender Sicht, Frankfurt a. M.; Ossenbühl, F. (2012): Frauenquote für Leitungsorgane von Privatunternehmen, in: Neue Juristische Wochenzeitschrift 65 (7), S. 417-422; Papier, H.-J./Heidebach M. (2011): Die Einführung einer gesetzlichen Frauenquote für Aufsichtsräte deutscher Unter- nehmen unter verfassungsrechtlichen Aspekten, in: Zeitschrift für Unternehmens- und Gesellschaftsrecht 40 (4), S. 305-333; Spindler, G./Brandt K. (2011): Verfassungsrechtliche Zulässigkeit einer Gleichstellungsquote im Aufsichtsrat der börsennotierten AG, in: Neue Zeitschrift für Gesellschaftsrecht 14 (11), S. 401-405.

22 A.A. BDA/BDI (2014), a.a.O., S. 9.

23 Vgl. Pütz, L. (2014): Zur Sache - Regierung muss bei Frauenquote nachbessern, in: Magazin Mitbestimmung 9/2014, S. 58f.; Pütz,L./Weckes, M. (2014), a.a.O.

24 Siehe hierzu auch DGB (2014a), a.a.O., S. 32, der in seiner Stellungnahme darauf hinwiest, "dass eine ausschließliche Anknüpfung der $30 \%-$ Geschlechterquote an die paritätische Mitbestimmung den bereits jetzt spürbaren Versuchen einzelner Unternehmen, der deutschen Unternehmensmitbestimmung durch die Nutzung europäischer Rechtsformen zu entgehen, Vorschub leistet." 
genüber den Anteilseignern vorliegt. ${ }^{25}$ Diese Ungleichbehandlung wird dadurch verstärkt, dass sich die Arbeitnehmerseite laut $\$ 7$ Abs. 2 i.V.m. $\$ 15$ Abs. 1 MitbestG aus verschiedenen Gruppen zusammensetzt, weshalb einzelne Gruppen zum Teil eine deutlich über $30 \%$ liegende Quote erfüllen müssten. Zwar wird mittelfristig nicht argumentiert werden können, dass zu wenige Frauen in den Betrieben beschäftigt sind, um geeignete Kandidatinnen zu finden. Denn der Vorschlag sieht vor, dass z. B. beim MitbestG bei 2.000-10.000 Arbeitnehmern eine und erst ab in der Regel mehr als 10.000 Arbeitnehmer zwei Frauen als betriebliche Vertreter zu wählen sind. Eine Ungleichbehandlung liegt, aufgrund der starren Quote und der Tatsache, dass der Leitende Angestellte nicht quotiert werden kann, indes trotzdem vor. ${ }^{26}$ Hieraus wiederum resultiert die Gefahr, dass die notwendige Quote nicht die hinreichende Unterstützung der Belegschaft bekommt. Es ist schon schwer vermittelbar, dass die Leitenden Angestellten einen Platz im Aufsichtsrat haben, obwohl sie eine absolute Minderheit der Belegschaft stellen. Dass sie zukünftig aber auch noch bei der Geschlechterquote unberücksichtigt bleiben, und zwar, obwohl gerade die Erhöhung des Frauenanteils im Management - also auch bei den Leitenden Angestellten - Ziel des Gesetzes ist, erscheint für viele Arbeitnehmer nicht nachvollziehbar.

Auch die Soll-Regelung zur Europäischen Aktiengesellschaft (SE) sowie zur grenzüberschreitenden Verschmelzung überzeugt nicht. Wird den Anteilseignern im Aufsichtsorgan einer SE die Quote nicht abgefordert, was möglich wäre, und bleibt es bei dem Abgrenzungskriterium „voll mitbestimmt", besteht die Gefahr, dass die SE zur Vermeidung der „Frauenquote“ und damit zusätzlich auch zur Vermeidung der Mitbestimmung genutzt wird. Der RefE fokussiert sich letztlich zu stark auf die Frage der Geschlechtergerechtigkeit und vernachlässigt die wichtige Funktion der Mitbestimmung für das deutsche System der Corporate Governance.

\section{Fazit}

Erklärungen des $\mathrm{DGB}^{27}$ zeigen, dass sich die Gewerkschaften ihrer Verantwortung für die Geschlechtergerechtigkeit bewusst sind und ihnen eine gelungene Ausgestaltung eines solchen Gesetzes wichtig ist. Das zeigt auch die bisherige Besetzung der Aufsichtsratsmandate auf Arbeitnehmerseite. Im anstehenden Gesetzgebungsprozess sollte daher die Chance genutzt werden, an den oben genannten Punkten nachzubessern. Hierzu zählt insbesondere, dass die Einführung der Quote ab 2.000 Arbeitnehmern plausibler ist, statt diese an das Merkmal „voll mitbestimmt“ zu koppeln. Die Gleichberechtigung der Geschlechter ist ein gesamtgesellschaftliches Anliegen, das nicht auf eine Mitbestimmungsform im Aufsichtsrat reduziert werden sollte. Auch sollte für Unternehmen in der Rechtsform einer SE ebenso die Geschlechterquote gelten. Zwar kann den Arbeitnehmervertretern im Aufsichtsrat aufgrund des zurzeit geltenden europäischen Rechts dies nicht (verpflichtend) abverlangt werden. Für die Anteilseigner im Aufsichtsorgan einer SE ist die Quote aber unproblematisch regelbar.

Besonders wichtig ist zudem, dass die demokratische Legitimation der Arbeitnehmervertreter bestehen bleibt. ${ }^{28}$ Jede Quotenregelung führt auch dazu, dass der Wählerwille „verfälscht“ wird. Daher ist Fingerspitzengefühl gefragt. Das Legitimationsband zwischen der Belegschaft, den Wählern und ihren Vertretern im Aufsichtsrat darf nicht durchtrennt werden. Um dieses zu gewährleisten, bedarf es aber mehr als gesetzlicher Regelungen. Es erfordert eine gesellschaftliche Diskussion und weitere (Übergangs-)Lösungen, die den Argwohn vor der Quote nehmen und ein Umdenken befördern. Geschlechtergerechtigkeit ist mehr als eine Quote für den Aufsichtsrat.
25 Vgl. DGB (2014a), a.a.O., S. 33, der aufgrund dieser Ungleichbehandlung andere Lösungen „empfiehlt“.

$26 \mathrm{Ob}$ man diese Ungleichbehandlung vor dem Hintergrund, dass die Verfassungsmäßigkeit einer festen Quote noch nicht geklärt ist (vgl. Fn. 21), aufrechterhalten will, sollte sich der Gesetzgeber überlegen.

27 Vgl. DGB (2014a), a.a.O. und DGB (2014b): Positionspapier des Bundesvorstands des Deutschen Gewerkschaftsbundes: Für gesetzliche Regelungen zur Durchsetzung einer Geschlechterquote in Aufsichtsräten.

28 Vgl. zur Abschwächung der demokratischen Legitimation durch die Sanktion des leeren Stuhls ULA (2014), a.a.O. und DGB (2014a), a.a.O.

\section{AUTOREN}

LASSE PÜTZ, Rechtsanwalt, ist Referatsleiter Wirtschaftsrecht in der Hans-Böckler-Stiftung. Arbeitsschwerpunkte: Unternehmensrecht, Unternehmensmitbestimmung, europäisches Gesellschaftsrecht, Corporate Governance, Compliance.

lasse-puetz@boeckler.de

MARION WECKES, Dipl. Volkswirtin, ist Referatsleiterin Wirtschaft in der Hans-Böckler-Stiftung. Arbeitsschwerpunkte: Jahresabschlussanalyse, Vorstandsvergütung, Mitarbeiterkapitalbeteiligung, Aktienrückkauf, Frauen in Führungspositionen.

marion-weckes@boeckler.de 